\title{
On Fundamental Solutions in Clifford Analysis
}

\author{
F. Brackx*, H. De Schepper*, M.E. Luna-Elizarrarás ${ }^{\ddagger}$ and M. Shapiro ${ }^{\ddagger}$ \\ * Clifford Research Group, Faculty of Engineering, Ghent University, Belgium \\ * Instituto Politécnico Nacional, Mexico City, Mexico
}

\begin{abstract}
Euclidean Clifford analysis is a higher dimensional function theory offering a refinement of classical harmonic analysis. The theory is centred around the concept of monogenic functions, which constitute the kernel of a first order vector valued, rotation invariant, differential operator $\underline{\partial}$ called the Dirac operator, which factorizes the Laplacian. More recently, Hermitean Clifford analysis has emerged as a new branch of Clifford analysis, offering yet a refinement of the Euclidean case; it focusses on a subclass of monogenic functions, i.e. the simultaneous null solutions, called Hermitean (or $\mathrm{h}^{-}$) monogenic functions, of two Hermitean Dirac operators $\partial_{z}$ and $\partial_{z^{\dagger}}$ which are invariant under the action of the unitary group, and constitute a splitting of the original Euclidean Dirac operator. In Euclidean Clifford analysis, the Clifford-Cauchy integral formula has proven to be a corner stone of the function theory, as is the case for the traditional Cauchy formula for holomorphic functions in the complex plane. Also a Hermitean Clifford-Cauchy integral formula has been established by means of a matrix approach. Naturally Cauchy integral formulae rely upon the existence of fundamental solutions of the Dirac operators under consideration. The aim of this paper is twofold. We want to reveal the underlying structure of these fundamental solutions and to show the particular results hidden behind a formula such as e.g. $\underline{\partial} E=\delta$. Moreover we will refine these relations by constructing fundamental solutions for the differential operators issuing from the Euclidean and Hermitean Dirac operators by splitting the Clifford algebra product into its dot and wedge parts.
\end{abstract}

\section{Introduction}

The Cauchy integral formula for holomorphic functions in the complex plane may be generalized to several complex variables in two ways: either one takes a holomorphic kernel and an integral over the distinguished boundary $\partial_{0} \widetilde{D}=\prod_{j=1}^{n} \partial \widetilde{D}_{j}$ of a polydisk $\widetilde{D}=\prod_{j=1}^{n} \widetilde{D}_{j}$ in $\mathbb{C}^{n}$ :

$$
f\left(z_{1}, \ldots, z_{n}\right)=\frac{1}{(2 \pi i)^{n}} \int_{\partial_{0} \widetilde{D}} \frac{f\left(\xi_{1}, \ldots, \xi_{n}\right)}{\left(\xi_{1}-z_{1}\right) \cdots\left(\xi_{n}-z_{n}\right)} d \xi_{1} \wedge \cdots \wedge d \xi_{n}, \quad z_{j} \in \stackrel{\circ}{\widetilde{D}}_{j}
$$

or one takes an integral over the (piecewise) smooth boundary $\partial D$ of a bounded domain $D$ in $\mathbb{C}^{n}$ in combination with the Martinelli-Bochner kernel, see e.g. [26], which is not holomorphic anymore but still harmonic:

$$
f(z)=\int_{\partial D} f(\xi) U(\xi, z), \quad z \in \stackrel{\circ}{D}
$$

with

$U(\xi, z)=\frac{(n-1) !}{(2 \pi i)^{n}} \sum_{j=1}^{n}(-1)^{j-1} \frac{\xi_{j}^{c}-z_{j}^{c}}{|\xi-z|^{2 n}} d \xi_{1}^{c} \wedge \cdots \wedge d \xi_{j-1}^{c} \wedge d \xi_{j+1}^{c} \wedge \cdots \wedge d \xi_{n}^{c} \wedge d \xi_{1} \wedge \cdots \wedge d \xi_{n}$

where ${ }^{c}$ denotes the complex conjugate. The history of formula (2), obtained independently by Martinelli and Bochner, has been described in detail in [25]. It reduces to the traditional Cauchy integral formula when $n=1$, while for $n>1$, it establishes a connection between harmonic and holomorphic functions. Of course the fact that the Martinelli-Bochner kernel is harmonic only, not holomorphic, and that, for $n>1$, and for an arbitrary sufficiently smooth 
domains, there are no integral representations with holomorphic kernel, becomes crucial for explaining the well-known differences between the theories in one and $n>1$ variables.

Another alternative for a generalization of the Cauchy integral formula is offered by Clifford analysis, where functions defined in Euclidean space $\mathbb{R}^{2 n} \cong \mathbb{C}^{n}$ and taking values in a Clifford algebra are considered. One focusses on so-called monogenic functions, i.e. null solutions of the elliptic Dirac operator $\partial_{\underline{X}}$ which is a square root of the Laplace operator: $\partial_{X}^{2}=-\Delta_{2 n}$. As the Dirac operator is rotation invariant, the name Euclidean Clifford analysis is used nowadays to refer to this setting. Standard references are [12, 21, 20, 24, 23]. In this framework the kernel appearing in the Clifford-Cauchy formula is monogenic, i.e., of the same class as the functions under consideration, up to a pointwise singularity, while the integral is taken over the complete boundary:

$$
f(\underline{X})=\int_{\partial D} E(\underline{\Xi}-\underline{X}) d \sigma_{\underline{\Xi}} f(\underline{\Xi}), \quad \underline{X} \in \stackrel{\circ}{D}
$$

with

$$
E(\underline{\Xi}-\underline{X})=\frac{1}{a_{2 n}} \frac{\bar{\Xi}-\underline{\bar{X}}}{|\underline{\Xi}-\underline{X}|^{2 n}},
$$

$a_{2 n}$ being the area of the unit sphere $S^{2 n-1}$ in $\mathbb{R}^{2 n} \cong \mathbb{C}^{n},-$ denoting the main conjugation in the Clifford algebra and $d \sigma_{\Xi}$ being a Clifford algebra valued differential form of order $(2 n-1)$ representing the oriented surface element. This Clifford-Cauchy integral formula is a corner stone in the development of the function theory. At its basis lies the fundamental solution $E(\underline{X})=\frac{1}{a_{2 n}} \frac{\bar{X}}{|\underline{X}|^{2 n}}$ of the Euclidean Dirac operator $\partial_{\underline{X}}$, which satisfies in distributional sense

$$
\partial_{\underline{X}} E(\underline{X})=\delta(\underline{X})
$$

where $\delta(\underline{X})$ is the Dirac distribution in $\mathbb{R}^{2 n}$.

In a series of recent papers, so-called Hermitean Clifford analysis has emerged as a refinement of the Euclidean case; it focusses on the simultaneous null solutions of the complex Hermitean Dirac operators $\partial_{\underline{z}}$ and $\partial_{\underline{z}^{\dagger}}$ which decompose the Euclidean Dirac operator and "factorize" the Laplace operator in the sense that $4\left(\partial_{\underline{z}}+\partial_{z^{\dagger}}\right)^{2}=4\left(\partial_{\underline{z}} \partial_{z^{\dagger}}+\partial_{z^{\dagger}} \partial_{\underline{z}}\right)=\Delta_{2 n}$. They are invariant under the action of the (special) unitary group. Although the study of complex Dirac operators was already initiated in $[28,27,29]$, a systematic development of the function theory in the Hermitean Clifford context, including the invariance properties with respect to the underlying Lie groups and Lie algebras, is still in full progress, see e.g. $[17,18,16,3,4,1,2,22,10]$. A Cauchy integral formula for Hermitean monogenic functions taking values in the complex Clifford algebra $\mathbb{C}_{2 n}$ is essential for further development of this function theory. A first result in this direction was obtained in [30], however for functions which are null solutions of only one of the Hermitean Dirac operators and moreover presenting a "fake" - as termed by the authors - Cauchy kernel, failing to be monogenic. In [11] a Cauchy integral formula for Hermitean monogenic functions has been established. However, from the start it was clear that the desired formula could not have the traditional form of (1) or (2). Indeed, it is known (see [4]) that in the special case where the functions considered do not take their values in the whole Clifford algebra $\mathbb{C}_{2 n}$, but in the $n$-homogeneous part $\mathbb{S}_{n}$ of complex spinor space $\mathbb{S}=\mathbb{C}_{2 n} I \cong \mathbb{C}_{n} I, I$ being a self-adjoint primitive idempotent, Hermitean monogenicity is equivalent with holomorphy in the underlying complex variables. It turned out that a matrix approach was the key to obtain the desired result. Moreover and as could be expected, the obtained Hermitean Cauchy integral formula reduces to the traditional Martinelli-Bochner formula (2) in the case of functions taking values in the particular part of complex spinor space mentioned. This also means that the theory of Hermitean monogenic functions not only refines Euclidean Clifford analysis (and thus harmonic analysis as well), but also has strong connections with the theory of functions of several complex variables, in some sense even encompassing it.

In this paper we will establish fundamental formulae underlying the key relation (3) and the similar ones for the Hermitean Dirac operators. Moreover we will construct fundamental solutions for the associated differential operators $\partial_{\underline{X}} \bullet, \partial_{\underline{X}} \wedge, \partial_{\underline{z}} \bullet, \partial_{\underline{z}} \wedge, \partial_{z^{\dagger}} \bullet, \partial_{z^{\dagger}} \wedge$, obtained by splitting the Clifford algebra or geometric product into its "dot" and "wedge" parts. 
These associated differential operators are the counterparts in the multivector language of Clifford analysis, of well-known differential operators for real and complex differential forms in Euclidean space, see [15, 19].

\section{Preliminaries of Clifford analysis}

The real Clifford algebra $\mathbb{R}_{0, m}$ is constructed over the vector space $\mathbb{R}^{0, m}$ endowed with a non-degenerate quadratic form of signature $(0, m)$ and generated by the orthonormal basis $\left(e_{1}, \ldots, e_{m}\right)$. The non-commutative Clifford or geometric multiplication in $\mathbb{R}_{0, m}$ is governed by the rules

$$
e_{\alpha} e_{\beta}+e_{\beta} e_{\alpha}=-2 \delta_{\alpha \beta}, \alpha, \beta=1, \ldots, m .
$$

As a basis for $\mathbb{R}_{0, m}$ one takes for any set $A=\left\{j_{1}, \ldots, j_{h}\right\} \subset\{1, \ldots, m\}$ the element $e_{A}=$ $e_{j_{1}} \ldots e_{j_{h}}$, with $1 \leq j_{1}<j_{2}<\cdots<j_{h} \leq m$, together with $e_{\emptyset}=1$, the identity element. Any Clifford number $a$ in $\mathbb{R}_{0, m}$ may thus be written as $a=\sum_{A} e_{A} a_{A}, a_{A} \in \mathbb{R}$, or still as $a=\sum_{k=0}^{m}[a]_{k}$, where $[a]_{k}=\sum_{|A|=k} e_{A} a_{A}$ is the so-called $k$-vector part of $a(k=0,1, \ldots, m)$. Euclidean space $\mathbb{R}^{0, m}$ is embedded in $\mathbb{R}_{0, m}$ by identifying $\left(X_{1}, \ldots, X_{m}\right)$ with the Clifford vector $\underline{X}=\sum_{\alpha=1}^{m} e_{\alpha} X_{\alpha}$, for which it holds that $\left.\underline{X}^{2}=-<\underline{X}, \underline{X}\right\rangle=-|\underline{X}|^{2}$. The Clifford or geometric product of two vectors splits into the sum of a scalar part, their commutative "dot" product, and a 2-vector, or bivector, part, their anti-commutative "wedge" product, viz

$$
\underline{X} \underline{Y}=\underline{X} \bullet \underline{Y}+\underline{X} \wedge \underline{Y}
$$

with

$$
\begin{aligned}
& \underline{X} \bullet \underline{Y}=\sum_{\alpha=1}^{m} X_{\alpha} Y_{\alpha}=\frac{1}{2}(\underline{X} \underline{Y}+\underline{Y} \underline{X}) \\
& \underline{X} \wedge \underline{Y}=\sum_{\alpha<\beta} e_{\alpha} e_{\beta}\left(X_{\alpha} Y_{\beta}-Y_{\alpha} X_{\beta}\right)=\frac{1}{2}(\underline{X} \underline{Y}-\underline{Y} \underline{X})
\end{aligned}
$$

The Fischer dual of the vector variable $\underline{X}$ is the vector valued first order differential operator $\partial_{\underline{X}}=\sum_{\alpha=1}^{m} e_{\alpha} \partial_{X_{\alpha}}$, called Dirac operator, underlying the notion of monogenicity of a function, a notion which is the higher dimensional counterpart of holomorphy in the complex plane. A function $f$ defined and differentiable in an open region $\Omega$ of $\mathbb{R}^{0, m}$ and taking values in $\mathbb{R}_{0, m}$ is called (left) monogenic in $\Omega$ if $\partial_{X}[f]=0$ in $\Omega$. As the Dirac operator is a square root of the Laplacian: $\Delta_{m}=-\partial_{\underline{X}}^{2}$, monogenicity can be regarded as a refinement of harmonicity. We refer to this setting as the Euclidean case, since the fundamental group leaving the Dirac operator $\partial_{\underline{X}}$ invariant is the special orthogonal group $\mathrm{SO}(m ; \mathbb{R})$, which is doubly covered by the $\operatorname{Spin}(m)$ group of the Clifford algebra $\mathbb{R}_{0, m}$. For this reason, the Dirac operator $\partial_{\underline{X}}$ and the concept of monogenicity are also called rotation invariant.

When allowing for complex constants and moreover taking the dimension to be even: $m=2 n$, the same generators $\left(e_{1}, \ldots, e_{2 n}\right)$, still satisfying the multiplication rules (4), produce the complex Clifford algebra $\mathbb{C}_{2 n}$, which is the complexification of the real Clifford algebra $\mathbb{R}_{0,2 n}$, i.e. $\mathbb{C}_{2 n}=\mathbb{R}_{0,2 n} \oplus i \mathbb{R}_{0,2 n}$. Any complex Clifford number $\lambda \in \mathbb{C}_{2 n}$ may thus be written as $\lambda=a+i b, a, b \in \mathbb{R}_{0,2 n}$, an observation leading to the definition of the Hermitean conjugation $\lambda^{\dagger}=(a+i b)^{\dagger}=\bar{a}-i \bar{b}$, where the bar notation stands for the usual Clifford conjugation in $\mathbb{R}_{0,2 n}$, i.e. the main anti-involution for which $\bar{e}_{\alpha}=-e_{\alpha}, \alpha=1, \ldots, 2 n$. This Hermitean conjugation also leads to a Hermitean inner product and its associated norm on $\mathbb{C}_{2 n}$, given by $(\lambda, \mu)=\left[\lambda^{\dagger} \mu\right]_{0}$ and $|\lambda|=\sqrt{\left[\lambda^{\dagger} \lambda\right]_{0}}=\left(\sum_{A}\left|\lambda_{A}\right|^{2}\right)^{1 / 2}$.

This is the framework for so-called Hermitean Clifford analysis, a refinement of Euclidean Clifford analysis. An elegant way of introducing this setting consists in considering a so-called complex structure, i.e. a specific $\mathrm{SO}(2 n ; \mathbb{R})$-element $J$ for which $J^{2}=\mathbf{- 1}$ (see $[3,4]$ ). Here, $J$ is chosen to act upon the generators $e_{1}, \ldots, e_{2 n}$ of the Clifford algebra as

$$
J\left[e_{j}\right]=-e_{n+j} \quad \text { and } \quad J\left[e_{n+j}\right]=e_{j}, \quad j=1, \ldots, n .
$$

With $J$ one may associate two projection operators $\frac{1}{2}(\mathbf{1} \pm i J)$ which produce the main objects of the Hermitean setting by acting upon the corresponding objects in the Euclidean framework. 
First of all, the so-called Witt basis elements $\left(\mathfrak{f}_{j}, \mathfrak{f}_{j}^{\dagger}\right)_{j=1}^{n}$ for $\mathbb{C}_{2 n}$ are obtained through the action of $\pm \frac{1}{2}(\mathbf{1} \pm i J)$ on the orthogonal basis elements $e_{\alpha}$ :

$$
\begin{aligned}
& \mathfrak{f}_{j}=\frac{1}{2}(\mathbf{1}+i J)\left[e_{j}\right]=\frac{1}{2}\left(e_{j}-i e_{n+j}\right), \quad j=1, \ldots, n, \\
& \mathfrak{f}_{j}^{\dagger}=-\frac{1}{2}(\mathbf{1}-i J)\left[e_{j}\right]=-\frac{1}{2}\left(e_{j}+i e_{n+j}\right), \quad j=1, \ldots, n .
\end{aligned}
$$

The Witt basis elements satisfy the respective Grassmann and duality identities

$$
\begin{aligned}
& \mathfrak{f}_{j} \mathfrak{f}_{k}+\mathfrak{f}_{k} \mathfrak{f}_{j}=\mathfrak{f}_{j}^{\dagger} \mathfrak{f}_{k}^{\dagger}+\mathfrak{f}_{k}^{\dagger} \mathfrak{f}_{j}^{\dagger}=0, j, k=1, \ldots, n, \\
& \mathfrak{f}_{j} \mathfrak{f}_{k}^{\dagger}+\mathfrak{f}_{k}^{\dagger} \mathfrak{f}_{j}=\delta_{j k}, \quad j, k=1, \ldots, n .
\end{aligned}
$$

A vector $\underline{X}=\left(X_{1}, \ldots, X_{2 n}\right)$ in $\mathbb{R}^{0,2 n}$ is now denoted by $\left(x_{1}, \ldots, x_{n}, y_{1}, \ldots, y_{n}\right)$ and identified with the Clifford vector $\underline{X}=\sum_{j=1}^{n}\left(e_{j} x_{j}+e_{n+j} y_{j}\right)$, the action of the complex structure $J$ on $\underline{X}$ yielding the so-called twisted vector $\underline{X} \mid=J[\underline{X}]=\sum_{j=1}^{n}\left(e_{j} y_{j}-e_{n+j} x_{j}\right)$. The Clifford vectors $\underline{X}$ and $\underline{X}$ | anti-commute, since the vectors $\underline{X}$ and $\underline{X} \mid$ are orthogonal w.r.t. the standard Euclidean scalar product, a property which is formulated more precisely in the following lemma.

Lemma 1 One has

(i) $\underline{X} \bullet \underline{X} \mid=0$,

(ii) $\underline{X} \wedge \underline{X} \mid=\sum_{j \neq k} x_{j} y_{k}\left(e_{j} e_{k}-e_{n+k} e_{n+j}\right)-\sum_{j, k} e_{j} e_{n+k}\left(x_{j} x_{k}+y_{j} y_{k}\right)$,

(iii) $\underline{X} \mid \wedge \underline{X}=\sum_{j \neq k} x_{j} y_{k}\left(e_{k} e_{j}-e_{n+j} e_{n+k}\right)-\sum_{j, k} e_{n+k} e_{j}\left(x_{j} x_{k}+y_{j} y_{k}\right)$,

(iv) $\underline{X} \underline{X}|+\underline{X}| \underline{X}=\underline{X} \wedge \underline{X}|+\underline{X}| \wedge \underline{X}=0$.

The actions of the projection operators on the Clifford vector $\underline{X}$ then produce the conjugate Hermitean Clifford variables $\underline{z}$ and $\underline{z}^{\dagger}$ :

$$
\begin{aligned}
& \underline{z}=\frac{1}{2}(\mathbf{1}+i J)[\underline{X}]=\frac{1}{2}(\underline{X}+i \underline{X} \mid), \\
& \underline{z}^{\dagger}=-\frac{1}{2}(\mathbf{1}-i J)[\underline{X}]=-\frac{1}{2}(\underline{X}-i \underline{X} \mid),
\end{aligned}
$$

which may also be rewritten in terms of the Witt basis elements as

$$
\underline{z}=\sum_{j=1}^{n} \mathfrak{f}_{j} z_{j} \quad \text { and } \quad \underline{z}^{\dagger}=(\underline{z})^{\dagger}=\sum_{j=1}^{n} \mathfrak{f}_{j}^{\dagger} z_{j}^{c},
$$

where $n$ complex variables $z_{j}=x_{j}+i y_{j}$ have been introduced, with complex conjugates $z_{j}^{c}=x_{j}-i y_{j}, j=1, \ldots, n$. Finally, the Hermitean Dirac operators $\partial_{\underline{z}}$ and $\partial_{\underline{z}^{\dagger}}$ are obtained from the Euclidean Dirac operator $\partial_{\underline{X}}$ :

$$
\begin{aligned}
& \partial_{\underline{z}}^{\dagger}= \frac{1}{4}(\mathbf{1}+i J)\left[\partial_{\underline{X}}\right]=\frac{1}{4}\left(\partial_{\underline{X}}+i \partial_{\underline{X} \mid}\right), \\
& \partial_{\underline{z}}=-\frac{1}{4}(\mathbf{1}-i J)\left[\partial_{\underline{X}}\right]=-\frac{1}{4}\left(\partial_{\underline{X}}-i \partial_{\underline{X} \mid}\right),
\end{aligned}
$$

where also the so-called twisted Dirac operator $\partial_{\underline{X} \mid}=J\left[\partial_{\underline{X}}\right]=\sum_{j=1}^{n}\left(e_{j} \partial_{y_{j}}-e_{n+j} \partial_{x_{j}}\right)$ arises, to which a notion of monogenicity may be associated in a natural way as well. In terms of the Witt basis, the Hermitean Dirac operators are expressed as

$$
\partial_{\underline{z}}=\sum_{j=1}^{n} \mathfrak{f}_{j}^{\dagger} \partial_{z_{j}} \quad \text { and } \quad \partial_{\underline{z}^{\dagger}}=\left(\partial_{\underline{z}}\right)^{\dagger}=\sum_{j=1}^{n} \mathfrak{f}_{j} \partial_{z_{j}^{c}}
$$

involving the classical Cauchy-Riemann operators $\partial_{z_{j}^{c}}=\frac{1}{2}\left(\partial_{x_{j}}+i \partial_{y_{j}}\right)$ and their complex conjugates $\partial_{z_{j}}=\frac{1}{2}\left(\partial_{x_{j}}-i \partial_{y_{j}}\right)$ in the complex $z_{j}$-planes, $j=1, \ldots, n$.

The Hermitean vector variables and Dirac operators are isotropic, i.e.

$$
(\underline{z})^{2}=\left(\underline{z}^{\dagger}\right)^{2}=0 \quad \text { and } \quad\left(\partial_{\underline{z}}\right)^{2}=\left(\partial_{\underline{z}}^{\dagger}\right)^{2}=0
$$


whence the Laplacian $\Delta_{2 n}=-\partial_{\underline{X}}^{2}=-\partial_{\underline{X}}^{2}$ allows for the "factorization"

$$
\Delta_{2 n}=4\left(\partial_{\underline{z}} \partial_{\underline{z}^{\dagger}}+\partial_{\underline{z}^{\dagger}} \partial_{\underline{z}}\right)=4\left(\partial_{\underline{z}}+\partial_{\underline{z}^{\dagger}}\right)^{2}
$$

while also

$$
\left(\underline{z}+\underline{z}^{\dagger}\right)^{2}=\underline{z} \underline{z}^{\dagger}+\underline{z}^{\dagger} \underline{z}=|\underline{z}|^{2}=\left|\underline{z}^{\dagger}\right|^{2}=|\underline{X}|^{2}=|\underline{X}|^{2} .
$$

A continuously differentiable function $g$ on an open region $\Omega$ of $\mathbb{R}^{2 n}$ with values in the complex Clifford algebra $\mathbb{C}_{2 n}$ is called (left) Hermitean monogenic (or h-monogenic) in $\Omega$ if

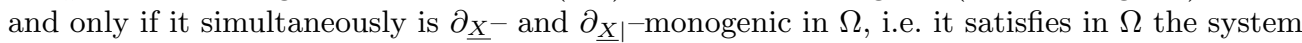

$$
\partial_{\underline{X}} g=0=\partial_{\underline{X} \mid} g
$$

or the equivalent system

$$
\partial_{\underline{z}} g=0=\partial_{\underline{z}^{\dagger}} g
$$

It remains to recall the group invariance underlying this system. To this end we consider the group $\widetilde{\mathrm{U}}(n) \subset \operatorname{Spin}(2 n)$, given by

$$
\widetilde{\mathrm{U}}(n)=\{s \in \operatorname{Spin}(2 n) \mid \exists \theta \geq 0: \bar{s} I=\exp (-i \theta) I\}
$$

its definition involving the self-adjoint primitive idempotent

$$
I=I_{1} \ldots I_{n}
$$

with $I_{j}=\mathfrak{f}_{j} \mathfrak{f}_{j}^{\dagger}=\frac{1}{2}\left(1-i e_{j} e_{n+j}\right), j=1, \ldots, n$. It has been proved, see [18], that this group constitutes a realisation in the Clifford algebra of the unitary group $\mathrm{U}(n)$, and moreover, that its associated action indeed leaves the Hermitean Dirac operators invariant. Less precisely, one thus says that these operators are invariant under the action of the unitary group, and so is the notion of $\mathrm{h}-$ monogenicity.

\section{The Euclidean Dirac operators}

It is well-known in Clifford analysis that the fundamental solutions of the Euclidean Dirac operators $\partial_{\underline{X}}$ and $\partial_{\underline{X}}$ are respectively given by

$$
E(\underline{X})=\frac{1}{a_{m}} \frac{\bar{X}}{|\underline{X}|^{m}}=-\frac{1}{a_{m}} \frac{\underline{X}}{r^{m}}
$$

and

$$
E \mid(\underline{X})=\frac{1}{a_{m}} \frac{\bar{X} \mid}{|\underline{X}|^{m}}=-\frac{1}{a_{m}} \frac{\underline{X} \mid}{r^{m}}
$$

where $a_{m}=\frac{2 \pi^{m / 2}}{\Gamma(m / 2)}$ is the area of the unit sphere $S^{m-1}$ in $\mathbb{R}^{m}$. Explicitly, this means that in distributional sense $\partial_{\underline{X}} E(\underline{X})=\delta(\underline{X})$ and $\partial_{\underline{X}} E \mid(\underline{X})=\delta(\underline{X} \mid)=\delta(\underline{X})$, where $\delta$ stands for the Dirac distribution in $\mathbb{R}^{2 n}$. As a matter of fact this result is quite remarkable since the Euclidean Dirac operators and their corresponding fundamental solutions are vector-valued, implying that the respective actions of $\partial_{\underline{X}}$ on $E$ and of $\partial_{\underline{X}}$ on $E \mid$ are expected to be parabivector-valued, i.e. resulting in a sum of a scalar and a bivector part. That the bivector parts indeed vanish only becomes clear when writing out both functions in terms of their components, i.e.

$$
\begin{aligned}
E(\underline{X}) & =\sum_{\alpha=1}^{m} E_{\alpha} e_{\alpha} \\
& =\sum_{j=1}^{n}\left(-\frac{1}{a_{2 n}} \frac{x_{j}}{r^{2 n}}\right) e_{j}+\left(-\frac{1}{a_{2 n}} \frac{y_{j}}{r^{2 n}}\right) e_{n+j}=\sum_{j=1}^{n} E_{j} e_{j}+E_{n+j} e_{n+j}, \\
E \mid(\underline{X}) & =\left.\sum_{\alpha=1}^{m} E\right|_{\alpha} e_{\alpha} \\
& =\sum_{j=1}^{n}\left(-\frac{1}{a_{2 n}} \frac{y_{j}}{r^{2 n}}\right) e_{j}-\left(-\frac{1}{a_{2 n}} \frac{x_{j}}{r^{2 n}}\right) e_{n+j}=\sum_{j=1}^{n} E_{n+j} e_{j}-E_{j} e_{n+j},
\end{aligned}
$$


and executing the calculations of distributional derivatives on the level of the components $E_{\alpha}$ and $\left.E\right|_{\alpha}, \alpha=1, \ldots, m$. The resulting expressions, summarised in the following lemma, involve the multi-dimensional Fp-distributions Finite Part which are defined using the well-known Finite Part-distribution on the real line; for a thorough study of these and related families of distributions in the framework of Clifford analysis we refer the reader to $[13,14,5,6,7,8,9]$.

Lemma 2 In distributional sense one has
(i) $\partial_{x_{j}} E_{j}=-\frac{1}{2 n} \delta-\frac{1}{a_{2 n}} F p \frac{1}{r^{2 n}}+\frac{2 n}{a_{2 n}} F p \frac{x_{j}^{2}}{r^{2 n+2}}, \quad j=1, \ldots, n$;
(ii) $\partial_{y_{j}} E_{n+j}=-\frac{1}{2 n} \delta-\frac{1}{a_{2 n}} F p \frac{1}{r^{2 n}}+\frac{2 n}{a_{2 n}} F p \frac{y_{j}^{2}}{r^{2 n+2}}, \quad j=1, \ldots, n$;
(iii) $\partial_{x_{k}} E_{j}=\frac{2 n}{a_{2 n}} F p \frac{x_{k} x_{j}}{r^{2 n+2}}, \quad k \neq j=1, \ldots, n$;
(iv) $\partial_{y_{k}} E_{n+j}=\frac{2 n}{a_{2 n}} F p \frac{y_{k} y_{j}}{r^{2 n+2}}, \quad k \neq j=1, \ldots, n$;
(v) $\partial_{x_{k}} E_{n+j}=\frac{2 n}{a_{2 n}} F p \frac{x_{k} y_{j}}{r^{2 n+2}}, \quad k, j=1, \ldots, n$;
(vi) $\partial_{y_{k}} E_{j}=\frac{2 n}{a_{2 n}} F p \frac{y_{k} x_{j}}{r^{2 n+2}}, \quad k, j=1, \ldots, n$.

Invoking the results obtained in Lemma 2, a direct calculation then yields the respective actions of $\partial_{\underline{X}}$ and $\partial_{\underline{X}} \mid$ on $E$ and $E \mid$. Note that, in particular, (i) and (ii) below were already obtained in [12], however through other considerations.

Proposition 1 In distributional sense one has

(i) $\partial_{\underline{X}} E=\delta$;

(ii) $\partial_{\underline{X} \mid} E \mid=\delta$;

(iii) $\partial_{\underline{X}} E \mid=\left(\sum_{j=1}^{n} e_{j} e_{n+j}\right)\left(\frac{1}{n} \delta+\frac{2}{a_{2 n}} F p \frac{1}{r^{2 n}}\right)+\frac{2 n}{a_{2 n}} F p \frac{X X}{r^{2 n+2}}$;

(iv) $\partial_{\underline{X} \mid} E=-\left(\sum_{j=1}^{n} e_{j} e_{n+j}\right)\left(\frac{1}{n} \delta+\frac{2}{a_{2 n}} F p \frac{1}{r^{2 n}}\right)+\frac{2 n}{a_{2 n}} F p \frac{\underline{X} \mid \underline{X}}{r^{2 n+2}}$;

(v) $\partial_{\underline{X}} E \mid+\partial_{\underline{X}} E=0$.

Proof.

We first calculate (i):

$$
\begin{aligned}
& \partial_{\underline{X}} E(\underline{X})= \sum_{k=1}^{n}\left(e_{k} \partial_{x_{k}}+e_{n+k} \partial_{y_{k}}\right)\left[\sum_{j=1}^{n} e_{j} E_{j}+e_{n+j} E_{n+j}\right] \\
&=- \sum_{j=1}^{n}\left(\partial_{x_{j}} E_{j}+\partial_{y_{j}} E_{n+j}\right)+\sum_{j<k} e_{k} e_{j}(\underbrace{\partial_{x_{k}} E_{j}-\partial_{x_{j}} E_{k}}_{0}) \\
&+\sum_{j<k} e_{n+k} e_{n+j}(\underbrace{\partial_{y_{k}} E_{n+j}-\partial_{y_{j}} E_{n+k}}_{0}) \\
&+\sum_{j} \sum_{k} e_{k} e_{n+j} \partial_{x_{k}} E_{n+j}+\sum_{j} \sum_{k} e_{n+k} e_{j} \partial_{y_{k}} E_{j} \\
&=- \sum_{j=1}^{n}\left(\partial_{x_{j}} E_{j}+\partial_{y_{j}} E_{n+j}\right)+\sum_{j} \sum_{k} e_{k} e_{n+j}(\underbrace{\partial_{x_{k}} E_{n+j}-\partial_{y_{j}} E_{k}}_{0}) \\
&=- \sum_{j=1}^{n}\left(-\frac{2}{2 n} \delta-\frac{2}{a_{2 n}} F_{p} \frac{1}{r^{2 n}}+\frac{2 n}{a_{2 n}} F_{p} \frac{x_{j}^{2}+y_{j}^{2}}{r^{2 n+2}}\right) \\
&=\delta+\frac{2 n}{a_{2 n}} F_{p} \frac{1}{r^{2 n}}-\frac{2 n}{a_{2 n}} F_{p} \frac{\sum x_{j}^{2}+y_{j}^{2}}{r^{2 n+2}} \\
&=\delta
\end{aligned}
$$


In a similar way, (ii),(iii) and (iv) are obtained. Finally, (v) follows by adding (iii) and (iv) and taking Lemma 2(iv) into account.

The above results may now be refined by splitting the Clifford algebra product - which is in fact always tacitly assumed when a Clifford differential operator acts on a Clifford algebra valued function or distribution - into its dot and wedge parts . The resulting operators

$$
\begin{array}{lll}
\partial_{\underline{X}} \wedge & =\sum_{\alpha=1}^{m} \partial_{X_{\alpha}} e_{\alpha} \wedge, & \partial_{\underline{X} \mid} \wedge=\sum_{\alpha=1}^{m} \partial_{\left.X\right|_{\alpha}} e_{\alpha} \wedge \\
\partial_{\underline{X}} \bullet & =\sum_{\alpha=1}^{m} \partial_{X_{\alpha}} e_{\alpha} \bullet \quad, \quad \partial_{\underline{X} \mid} \wedge=\sum_{\alpha=1}^{m} \partial_{\left.X\right|_{\alpha}} e_{\alpha} \bullet
\end{array}
$$

may be identified with the well known $d$ and $d^{*}$ operators of exterior derivative and coderivative (and their twisted counterparts $d \mid$ and $\left.d\right|^{*}$ ) of smooth differential forms in Euclidean space. For a detailed overview of the similarities between real and complex differential forms in open subsets of $\mathbb{R}^{m}$ and $\mathbb{C}^{n}$ on the one hand and multivector functions in Euclidean and Hermitean Clifford analysis on the other hand, we refer the reader to $[15,19]$.

Either by direct computation using the results of Lemma 2, or by identifying the scalar and bivector parts in the relations contained in Proposition 1, the following relations are obtained.

Proposition 2 In distributional sense one has

(i) $\partial_{\underline{X}} \bullet E=\delta$;

(ii) $\partial_{\underline{X}} \wedge E=0$;

(iii) $\partial_{\underline{X}} \bullet E \mid=\delta$;

(iv) $\partial_{\underline{X} \mid} \wedge E \mid=0$;

(v) $\partial_{\underline{X}} \bullet E \mid=0$;

(vi) $\partial_{\underline{X}} \wedge E \mid=\left(\sum_{j=1}^{n} e_{j} e_{n+j}\right)\left(\frac{1}{n} \delta+\frac{2}{a_{2 n}} F p \frac{1}{r^{2 n}}\right)+\frac{2 n}{a_{2 n}} F p \frac{X X}{r^{2 n+2}}$;

(vii) $\partial_{\underline{X}} \bullet E=0$

(viii) $\partial_{\underline{X} \mid} \wedge E=-\left(\sum_{j=1}^{n} e_{j} e_{n+j}\right)\left(\frac{1}{n} \delta+\frac{2}{a_{2 n}} F p \frac{1}{r^{2 n}}\right)+\frac{2 n}{a_{2 n}} F p \frac{\underline{X} \mid \underline{X}}{r^{2 n+2}}$;

(ix) $\partial_{\underline{X}} \wedge E \mid+\partial_{\underline{X}} \wedge=E=0$.

Observe that the regular distributions $E$ and $E \mid$ turn out to be the fundamental solutions of the differential operators $\partial_{\underline{X}} \bullet$ and $\partial_{\underline{X}} \mid \bullet$, respectively, while the fundamental solutions of the operators $\partial_{\underline{X}} \wedge$ and $\partial_{\underline{X}} \wedge$, if any, are not known to us.

\section{The Hermitean Dirac operators}

Hermitean counterparts to the pair of fundamental solutions $(E, E \mid)$ of the Euclidean Dirac operators $\left(\partial_{\underline{X}}, \partial_{\underline{X}}\right)$ may be introduced as

$$
\mathcal{E}=-(E+i E \mid), \quad \mathcal{E}^{\dagger}=(E-i E \mid)
$$

or, explicitly:

$$
\begin{aligned}
\mathcal{E}(\underline{z}) & =\frac{2}{a_{2 n}} \frac{\underline{z}}{|\underline{z}|^{2 n}}=\sum_{j=1}^{n} \mathfrak{f}_{j}\left(\frac{2}{a_{2 n}} \frac{z_{j}}{r^{2 n}}\right)=\sum_{j=1}^{n} \mathfrak{f}_{j} \mathcal{E}_{j}, \\
\mathcal{E}^{\dagger}(\underline{z}) & =\frac{2}{a_{2 n}} \frac{\underline{z}^{\dagger}}{|\underline{z}|^{2 n}}=\sum_{j=1}^{n} \mathfrak{f}_{j}^{\dagger}\left(\frac{2}{a_{2 n}} \frac{z_{j}^{c}}{r^{2 n}}\right)=\sum_{j=1}^{n} \mathfrak{f}_{j}^{\dagger} \mathcal{E}_{j}^{c},
\end{aligned}
$$

where the meaning of $\mathcal{E}_{j}$ and $\mathcal{E}_{j}^{c},(j=1, \ldots, n)$ is obvious. However, these are not the fundamental solutions to the respective Hermitean Dirac operators $\partial_{\underline{z}}$ and $\partial_{z^{\dagger}}$, since, as was shown in [11], the following formulae hold. 


\section{Proposition 3 One has}

$$
\begin{aligned}
\partial_{\underline{z}} \mathcal{E}(\underline{z}) & =\frac{1}{n} \beta \delta\left(\underline{z}, \underline{z}^{\dagger}\right)+\frac{2}{a_{2 n}} \beta F p \frac{1}{r^{2 n}}-\frac{2}{a_{2 n}} n F p \frac{\underline{z}^{\dagger} \underline{z}}{r^{2 n+2}} ; \\
\partial_{\underline{z}^{\dagger}} \mathcal{E}(\underline{z}) & =0 \\
\partial_{\underline{z}} \mathcal{E}^{\dagger}(\underline{z}) & =0 \\
\partial_{\underline{z}^{\dagger}} \mathcal{E}^{\dagger}(\underline{z}) & =\frac{1}{n}(n-\beta) \delta\left(\underline{z}, \underline{z}^{\dagger}\right)+\frac{2}{a_{2 n}}(n-\beta) F p \frac{1}{r^{2 n}}-\frac{2}{a_{2 n}} n F p \frac{\underline{z} \underline{z}^{\dagger}}{r^{2 n+2}}
\end{aligned}
$$

where the para-bivector $\beta$, also called the spin-Euler operator, is given by $\beta=\sum_{j=1}^{n} \mathfrak{f}_{j}^{\dagger} \mathfrak{f}_{j}=$ $\frac{n}{2}+\frac{i}{2} \sum_{j=1}^{n} e_{j} e_{n+j}$.

Again these results may be refined by making the calculations componentwise, as well as by splitting the Clifford product into its dot and wedge parts.

Lemma 3 For all $j \neq k=1, \ldots, n$ one has
(i) $\partial_{z_{j}} \mathcal{E}_{j}=\frac{1}{n} \delta+\frac{2}{a_{2 n}} F p \frac{1}{r^{2 n}}-\frac{2 n}{a_{2 n}} F p \frac{\left|z_{j}\right|^{2}}{r^{2 n+2}}$;
(ii) $\partial_{z_{k}} \mathcal{E}_{j}=-\frac{2 n}{a_{2 n}} F p \frac{z_{j} z_{k}^{c}}{r^{2 n+2}}$;
(iii) $\partial_{z_{j}^{c}} \mathcal{E}_{j}^{c}=\frac{1}{n} \delta+\frac{2}{a_{2 n}} F p \frac{1}{r^{2 n}}-\frac{2 n}{a_{2 n}} F p \frac{\left|z_{j}\right|^{2}}{r^{2 n+2}}$;
(iv) $\partial_{z_{k}^{c}} \mathcal{E}_{j}^{c}=-\frac{2 n}{a_{2 n}} F p \frac{z_{k} z_{j}^{c}}{r^{2 n+2}}$;
(v) $\partial_{z_{k}} \mathcal{E}_{j}^{c}=\partial_{z_{j}} \mathcal{E}_{k}^{c}=-\frac{2 n}{a_{2 n}} F p \frac{z_{j}^{c} z_{k}^{c}}{r^{2 n+2}}$;
(vi) $\partial_{z_{k}^{c}} \mathcal{E}_{j}=\partial_{z_{j}^{c}} \mathcal{E}_{k}=-\frac{2 n}{a_{2 n}} F p \frac{z_{j} z_{k}}{r^{2 n+2}}$.

Proposition 4 One has

$$
\begin{aligned}
& \text { (i) } \partial_{\underline{z}} \bullet \mathcal{E}=\frac{1}{2} \delta ; \\
& \text { (ii) } \partial_{\underline{z}} \wedge \mathcal{E}=\sum_{j} \mathfrak{f}_{j}^{\dagger} \wedge \mathfrak{f}_{j}\left(\frac{1}{n} \delta+\frac{2}{a_{2 n}} F p \frac{1}{r^{2} n}\right)-\sum_{j, k} \mathfrak{f}_{k}^{\dagger} \wedge \mathfrak{f}_{j}\left(\frac{2 n}{a_{2 n}} F p \frac{z_{j} z_{k}^{c}}{r^{2 n+2}}\right) \\
& \text { (iii) } \partial_{\underline{z}^{\dagger}} \bullet \mathcal{E}^{\dagger}=\frac{1}{2} \delta ; \\
& \text { (iv) } \partial_{\underline{z}^{\dagger}} \wedge \mathcal{E}^{\dagger}=\sum_{j} \mathfrak{f}_{j} \wedge \mathfrak{f}_{j}^{\dagger}\left(\frac{1}{n} \delta+\frac{2}{a_{2 n}} F p \frac{1}{r^{2} n}\right)-\sum_{j, k} \mathfrak{f}_{k} \wedge \mathfrak{f}_{j}^{\dagger}\left(\frac{2 n}{a_{2 n}} F p \frac{z_{k} z_{j}^{c}}{r^{2 n+2}}\right) \\
& \text { (v) } \partial_{\underline{z}} \bullet \mathcal{E}^{\dagger}=0 \\
& \text { (vi) } \partial_{\underline{z}} \wedge \mathcal{E}^{\dagger}=0 \\
& \text { (vii) } \partial_{\underline{z}^{\dagger}} \bullet \mathcal{E}=0 \\
& \text { (viii) } \partial_{\underline{z}^{\dagger}} \wedge \mathcal{E}=0 .
\end{aligned}
$$

Proof.

We will first calculate (i) and (ii), respectively:

$$
\begin{aligned}
\partial_{\underline{z}} \bullet \mathcal{E} & =\sum_{j, k} \mathfrak{f}_{k}^{\dagger} \bullet \mathfrak{f}_{j} \partial_{z_{k}} \mathcal{E}_{j}=\sum_{j, k}\left(\frac{1}{2} \delta_{j k}\right) \partial_{z_{k}} \mathcal{E}_{j} \\
& =\frac{1}{2} \sum_{j} \partial_{z_{j}} \mathcal{E}_{j}=\frac{1}{2} \sum_{j}\left(\frac{1}{n} \delta+\frac{2}{a_{2 n}} \mathrm{Fp} \frac{1}{r^{2 n}}-\frac{2 n}{a_{2 n}} \mathrm{Fp} \frac{\left|z_{j}\right|^{2}}{r^{2 n+2}}\right)=\frac{1}{2} \delta ; \\
\partial_{\underline{z}} \wedge \mathcal{E} & =\sum_{j, k} \mathfrak{f}_{k}^{\dagger} \wedge \mathfrak{f}_{j} \partial_{z_{k}} \mathcal{E}_{j}=\sum_{j} \mathfrak{f}_{j}^{\dagger} \wedge \mathfrak{f}_{j} \partial_{z_{j}} \mathcal{E}_{j}+\sum_{j \neq k} \mathfrak{f}_{k}^{\dagger} \wedge \mathfrak{f}_{j} \partial_{z_{k}} \mathcal{E}_{j} \\
& =\sum_{j} \mathfrak{f}_{j}^{\dagger} \wedge \mathfrak{f}_{j}\left(\frac{1}{n} \delta+\frac{2}{a_{2 n}} \mathrm{Fp} \frac{1}{r^{2 n}}-\frac{2 n}{a_{2 n}} \mathrm{Fp} \frac{\left|z_{j}\right|^{2}}{r^{2 n+2}}\right)+\sum_{j \neq k} \mathfrak{f}_{k}^{\dagger} \wedge \mathfrak{f}_{j}\left(-\frac{2 n}{a_{2 n}} \mathrm{Fp} \frac{z_{j} z_{k}^{c}}{r^{2 n+2}}\right) \\
& =\sum_{j} \mathfrak{f}_{j}^{\dagger} \wedge \mathfrak{f}_{j}\left(\frac{1}{n} \delta+\frac{2}{a_{2 n}} \mathrm{Fp} \frac{1}{r^{2} n}\right)-\sum_{j, k} \mathfrak{f}_{k}^{\dagger} \wedge \mathfrak{f}_{j}\left(\frac{2 n}{a_{2 n}} \mathrm{Fp} \frac{z_{j} z_{k}^{c}}{r^{2 n+2}}\right) ;
\end{aligned}
$$


while noting that the computations for (iii) and (iv) proceed along similar lines. Next, we calculate (v) and (vi), yielding

$$
\partial_{\underline{z}} \bullet \mathcal{E}^{\dagger}=\sum_{j, k} \mathfrak{f}_{k}^{\dagger} \bullet \mathfrak{f}_{j}^{\dagger} \partial_{z_{k}} \mathcal{E}_{j}^{c}=0,
$$

since for all $j, k=1, \ldots, n$, the formula $\mathfrak{f}_{k}^{\dagger} \bullet \mathfrak{f}_{j}^{\dagger}=0$ holds and

$$
\partial_{\underline{z}} \wedge \mathcal{E}^{\dagger}=\sum_{j, k} \mathfrak{f}_{k}^{\dagger} \wedge \mathfrak{f}_{j}^{\dagger} \partial_{z_{k}} \mathcal{E}_{j}^{c}=\sum_{j \neq k} \mathfrak{f}_{k}^{\dagger} \wedge \mathfrak{f}_{j}^{\dagger} \partial_{z_{k}} \mathcal{E}_{j}^{c}=\sum_{j<k} \mathfrak{f}_{k}^{\dagger} \wedge \mathfrak{f}_{j}^{\dagger}\left(\partial_{z_{k}} \mathcal{E}_{j}^{c}-\partial_{z_{j}} \mathcal{E}_{k}^{c}\right)=0
$$

in view of Lemma $3(\mathrm{v})$. Again, the computations for (vii) and (viii) proceed along similar lines.

From the above results, it is readily seen that $2 \mathcal{E}(\underline{z})=\frac{4}{a_{2 n}} \frac{\underline{z}}{r^{2 n}}$ and $2 \mathcal{E}^{\dagger}(\underline{z})=\frac{4}{a_{2 n}} \frac{\underline{z}^{\dagger}}{r^{2 n}}$ are the fundamental solutions of the respective operators $\left(\partial_{\underline{z}} \bullet\right)$ and $\left(\partial_{\underline{z}^{\dagger}} \bullet\right)$. Furthermore, note that the operator $\partial_{z^{\dagger}} \wedge$ is the Hermitean Clifford analysis counterpart of the well-known differential operator "dee-bar" $\bar{\partial}$ in several complex variables theory (see [19]).

The results of Proposition 3 may now also be recovered by adding the appropriate formulae of the foregoing proposition. In particular one also finds:

$$
\begin{aligned}
\partial_{\underline{z}} \mathcal{E}+\partial_{\underline{z}^{\dagger}} \mathcal{E}^{\dagger}= & \partial_{\underline{z}} \bullet \mathcal{E}+\partial_{\underline{z}} \wedge \mathcal{E}+\partial_{\underline{z}^{\dagger}} \bullet \mathcal{E}^{\dagger}+\partial_{\underline{z}^{\dagger}} \wedge \mathcal{E}^{\dagger} \\
= & \frac{1}{2} \delta+\sum_{j} \mathfrak{f}_{j}^{\dagger} \wedge \mathfrak{f}_{j}\left(\frac{1}{n} \delta+\frac{2}{a_{2 n}} \mathrm{Fp} \frac{1}{r^{2} n}\right)-\sum_{j, k} \mathfrak{f}_{k}^{\dagger} \wedge \mathfrak{f}_{j}\left(\frac{2 n}{a_{2 n}} \mathrm{Fp} \frac{z_{j} z_{k}^{c}}{r^{2 n+2}}\right) \\
& +\frac{1}{2} \delta+\sum_{j} \mathfrak{f}_{j} \wedge \mathfrak{f}_{j}^{\dagger}\left(\frac{1}{n} \delta+\frac{2}{a_{2 n}} \mathrm{Fp} \frac{1}{r^{2} n}\right)-\sum_{j, k} \mathfrak{f}_{k} \wedge \mathfrak{f}_{j}^{\dagger}\left(\frac{2 n}{a_{2 n}} \mathrm{Fp} \frac{z_{k} z_{j}^{c}}{r^{2 n+2}}\right),
\end{aligned}
$$

which reduces to the fundamental formula $\partial_{\underline{z}} \mathcal{E}+\partial_{z^{\dagger}} \mathcal{E}^{\dagger}=\delta$ since the bivector terms simply cancel out!

Moreover, considering the particular circulant $(2 \times 2)$ matrices

$$
\mathcal{D}_{\left(\underline{z}, \underline{z}^{\dagger}\right)}=\left(\begin{array}{ll}
\partial_{\underline{z}} & \partial_{\underline{z}^{\dagger}} \\
\partial_{\underline{z}^{\dagger}} & \partial_{\underline{z}}
\end{array}\right), \quad \mathcal{E}=\left(\begin{array}{cc}
\mathcal{E} & \mathcal{E}^{\dagger} \\
\mathcal{E}^{\dagger} & \mathcal{E}
\end{array}\right), \quad \text { and } \quad \delta=\left(\begin{array}{cc}
\delta & 0 \\
0 & \delta
\end{array}\right),
$$

it is seen to hold that

$$
\mathcal{D}_{\left(\underline{z}, \underline{z}^{\dagger}\right)} \mathcal{E}(\underline{z})=\boldsymbol{\delta}(\underline{z})
$$

(see [11, 27], meaning that $\mathcal{E}$ may be considered as a fundamental solution of $\mathcal{D}_{\left(\underline{z}, \underline{z}^{\dagger}\right)}$. It is precisely this simple observation which has then lead us to the idea of a matrix approach to arrive at a Cauchy integral formula in the Hermitean setting (see [11]). Also note, as another remarkable fact, that the Dirac matrix $\mathcal{D}_{\left(\underline{z}, \underline{z}^{\dagger}\right)}$ factorizes the matrix Laplacian, since

$$
4 \mathcal{D}_{\left(\underline{z}, \underline{z}^{\dagger}\right)}\left(\mathcal{D}_{\left(\underline{z}, \underline{z}^{\dagger}\right)}\right)^{\dagger}=\left(\begin{array}{cc}
\Delta_{2 n} & 0 \\
0 & \Delta_{2 n}
\end{array}\right)=: \Delta_{2 n}
$$

a formula which is deeply similar to the factorization of the two-dimensional Laplace operator by the Cauchy-Riemann operator and its complex conjugate in the complex plane.

\section{References}

[1] R. Abreu Blaya, J. Bory Reyes, F. Brackx, B. De Knock, H. De Schepper, D. Pena Pena, F. Sommen, Hermitean Cauchy integral decomposition of continuous functions on hypersurfaces, Bound. Value Probl. 2009, 2009, ID 425256.

[2] R. Abreu Blaya, J. Bory Reyes, D. Pena Pena, F. Sommen, A boundary value problem for Hermitian monogenic functions, Bound. Value Probl. 2008, 2008, ID 385874. 
[3] F. Brackx , J. Bureš, H. De Schepper, D. Eelbode, F. Sommen and V. Souček, Fundaments of Hermitean Clifford Analysis. Part I: Complex structure, Compl. Anal. Oper. Theory 1(3), 2007, 341-365.

[4] F. Brackx, J. Bureš, H. De Schepper, D. Eelbode, F. Sommen and V. Souček, Fundaments of Hermitean Clifford Analysis. Part II: Splitting of h-monogenic equations, Complex Var. Elliptic Eq. 52(10-11), 2007, 1063-1079.

[5] F. Brackx, B. De Knock and H. De Schepper, A specific family of Clifford distributions. In: L. Son, W. Tutschke, S. Jain (eds.), Methods of complex and Clifford analysis, SAS Int. Publ., Delhi, 2004, 215-227.

[6] F. Brackx, B. De Knock and H. De Schepper, Multi-vector spherical monogenics, spherical means and distributions in Clifford analysis, Acta Math. Sin. 21(5), 2005, 1197-1208.

[7] F. Brackx, B. De Knock and H. De Schepper, On the Fourier spectra of distributions in Clifford analysis, Chin. Ann. Math. 27B(5), 2006, 495-506.

[8] F. Brackx, B. De Knock, H. De Schepper and D. Eelbode, A Calculus Scheme for Clifford Distributions, Tokyo J. Math. 29(2), 2006, 495-513.

[9] F. Brackx, B. De Knock, H. De Schepper and F. Sommen, Distributions in Clifford Analysis: an Overview. In: S.-L. Eriksson (ed.), Clifford Analysis and Applications Proceedings of the Summer School, Tampere 2004, Tampere University of Technology, Institute of Mathematics, Research Report No. 82, 2006, 59-73.

[10] F. Brackx, B. De Knock, H. De Schepper, A matrix Hilbert transform in Hermitean Clifford analysis, J. Math. Anal. Applic. 344(2), 2008, 1068-1078.

[11] F. Brackx, B. De Knock, H. De Schepper and F. Sommen, On Cauchy and MartinelliBochner integral formulae in Hermitean Clifford analysis, Bull. Braz. Math. Soc. (New Series) 40(3), 2009, 395-416.

[12] F. Brackx, R. Delanghe and F. Sommen, Clifford Analysis, Pitman Publishers, BostonLondon-Melbourne, 1982.

[13] F. Brackx, R. Delanghe and F. Sommen, Spherical means, distributions and convolution operators in Clifford analysis, Chin. Ann. Math. 24B(2), 2003, 133-146.

[14] F. Brackx, R. Delanghe and F. Sommen, Spherical means and distributions in Clifford analysis. In: T. Qian, T. Hempfling, A. McIntosh and F. Sommen (eds.), Advances in analysis and geometry: new developments using Clifford algebras, Trends in Mathematics, Birkhäuser Verlag, Basel-Boston-Berlin, 2004, 65-96.

[15] F. Brackx, R. Delanghe and F. Sommen, Differential forms and/or multivector functions, Cubo 7(2), 2005, 139-169.

[16] F. Brackx, H. De Schepper, D. Eelbode and V. Souček, The Howe dual pair in Hermitean Clifford analysis, accepted for publication in Rev. Mat. Iberoamericana.

[17] F. Brackx, H. De Schepper and F. Sommen, A theoretical framework for wavelet analysis in a Hermitean Clifford setting, Comm. Pure Appl. Anal. 6(3), 2007, 549-567.

[18] F. Brackx, H. De Schepper and F. Sommen, The Hermitian Clifford analysis toolbox, Appl. Clifford Algebras 18(3-4), 2008, 451-487.

[19] F. Brackx, H. De Schepper and V. Souček, Differential forms versus multi-vector functions in Hermitean Clifford analysis (to appear in $\mathrm{Cubo}$ ).

[20] F. Colombo, I. Sabadini, F. Sommen, D.C. Struppa, Analysis of Dirac Systems and Computational Algebra, Birkhäuser, Boston (2004).

[21] R. Delanghe, F. Sommen and V. Souček, Clifford Algebra and Spinor-Valued Functions, Kluwer Academic Publishers, Dordrecht, 1992.

[22] D. Eelbode, Irreducible $\mathfrak{s l}(m)$-modules of Hermitean monogenics, Complex Var. Elliptic Eq. 53(10), 2008, 975-987.

[23] J. Gilbert and M. Murray, Clifford Algebras and Dirac Operators in Harmonic Analysis, Cambridge University Press, Cambridge, 1991.

[24] K. Guerlebeck and W. Sproessig, Quaternionic and Clifford Calculus for Physicists and Engineers, Wiley, Chichester, 1998. 
[25] S. Krantz, Function theory of several complex variables, 2nd edition, Wadsworth \& Brooks/Cole, Pacific Grove, 1992.

[26] A. Kytmanov, The Bochner-Martinelli integral and its applications, Birkhaüser, BaselBoston-Berlin, 1995.

[27] R. Rocha-Chavez, M. Shapiro and F. Sommen, Integral theorems for functions and differential forms in $\mathbb{C}_{m}$, Research Notes in Math. 428, Chapman \& Hall/ CRC, New York, 2002.

[28] J. Ryan, Complexified Clifford analysis, Complex Variables: Theory \& Application 1 (1982), 119-149.

[29] I. Sabadini and F. Sommen, Hermitian Clifford analysis and resolutions, Math. Meth. Appl. Sci. 25 (16-18) (2002), 1395-1414.

[30] F. Sommen and D. Peña Peña, A Martinelli-Bochner formula for the Hermitian Dirac equation, Math. Meth. Appl. Sci. 30(9), 2007, 1049-1055. 